\title{
Multi- Spectrum Bands Allocation for Time-Varying Traffic in the Flexible Optical Network
}

\author{
KAMAGATE Beman Hamidja \\ Laboratoire de Recherche en Informatique et \\ Télécommunication (LARIT) \\ Ecole Doctorale Polytechnique de l'INP-HB \\ Yamoussoukro, Côte d'Ivoire \\ Michel BABRI \\ Laboratoire de Recherche en Informatique et \\ Télécommunication (LARIT) \\ INPH-HB \\ Yamoussoukro, Côte d’Ivoire
}

\author{
GOORE Bi Tra \\ Laboratoire de Recherche en Informatique et \\ Télécommunication (LARIT ) \\ INP-HB \\ Yamoussoukro, Côte d’Ivoire \\ Souleymane OUMTANAGA \\ Laboratoire de Recherche en Informatique et \\ Télécommunication (LARIT) \\ INP-HB \\ Yamoussoukro, Côte d’Ivoire
}

\begin{abstract}
The flexible optical networks are the promising solution to the exponential increase of traffic generated by telecommunications networks. They combine flexibility with the finest granularity of optical resources. Therefore, the flexible optical networks position themselves as a better solution than conventional WDM network. In the operational phase, traffic of connections fluctuates. In fact, the user's need is not the same during day periods. Such traffic may experiment evidence of rising working hours, end of months or years and decreases during the night or on holidays. This variation requires the expansion or contraction of the number of frequency slots allocated to a connection to match the exact needs of the moment. The expansion of the traffic around the reference frequency of connection may lead to blockage because it must share frequency slots with neighboring connections in compliance with the constraints of continuity, contiguity, and non-overlapping. In this study, we offer a technique for allocating frequency slots for time-varying traffic connections. We share out the additional traffic load on different spectrum paths by respecting the constraint of time synchronization related to the differential delay to reduce the blocking rate due to traffic fluctuation.
\end{abstract}

Keywords-Spectrum band; Multi-spectrum bands; timevarying traffic; elastic optical network

\section{INTRODUCTION}

The flexible optical network constitutes an efficient alternative facing the exponential rise of traffic. It compensates for the shortcomings of conventional WDM network which are the rigidity of the frequency spectrum and ressources waste. Based on the Optical-Orthogonal Frequency Division Multiplexing (O-OFDM) technology, it provides a finer granularity and allows the use of several parallel subcarriers or frequency slots for transmitting the traffic of a connection from a source node to a destination node on a definite physical path. The set defined by subcarriers and the physical path is the optical channel or spectrum path. The bandwidth of the optical channel is the sum of bandwidth of adjacent frequency slots that form it. Each frequency slot with a bandwidth typically set to $12.5 \mathrm{GHz}$. The number of frequency slots allocated to a connection depends on the flow of its traffic and transmission distance [1]. The allocation of frequency slots is subject to constraints. The first is the contiguity constraint, according to which frequency slots allocated to the same connection must be adjacent. The second constraint is the continuity of the frequency slots allocated to each link of the optical path, i.e., the utilization of the same frequency slots on all links which compose optical path, based on the assumption that there is no conversion. The last constraint is the constraint of non-overlapping, i.e.two different connections cannot simultaneously use the same frequency slots.

The management of these constraints and the multiplicity of frequency slots is a challenge in the determination of optimal spectrum path for a connection. That problem of optimal spectrum allocation is an Integer Linear Program (ILP) problem optimization. It includes two aspects that are the determination of physical path from the source to the destination of the connection and the determination of the frequency slots on that physical path which respects the constraints of flexible optical networks. Besides, this problem known as Routing and Spectrum Allocation (RSA) is reckoned as np-hard [2]. It is an extension of the well-known RWA problem in the conventional WDM networks [3]. The resolution of this problem is even more complex in the case of dynamic traffic variables in time. In fact, the fluctuating traffic of connection leads to resource sharing problems with neighboring connections. One has to ensure that different connections do not simultaneously seek the same frequency slots because it can cause the rejection of the request. This paper aims to contribute at solving that problem by developing a spectrum allocation mechanism which takes into account the possibility of sharing out traffic over different optical paths to reduce the blocking rate when the traffic of a connection undergoes a fluctuation. This mechanism is the multispectrum bands allocation; the frequency slots allocated to the connection are on different spectrum bands. A spectrum band 
is a portion of the spectrum consisted of several contiguous frequency slots. So here we exploit the possibility of distribution traffic among several spectrum bands of the different path by respecting the differential delay constraint due to Group Velocity Delay (GVD), propagation delay and latency in the nodes.

This study is structured as follows. Firstly Section II, we shall present the characteristics of flexible optical networks as well as solving approaches for time-varying traffic of slots at to varying dynamic traffic time base connection. Secondly, Section III is devoted to our contribution which is an approach based on multi-spectrum bands allocation. Thirdly in Section IV, we evaluate the performance of our proposal by comparing it to existent works. Then, we shall finish with a conclusion and provide perspectives for future works.

\section{SPECTRUM Allocation FOR Time-VARYING TRAFFiC IN FLEXIBLE OPTICAL NETWORKS}

\section{A. Feature of flexible optical networks}

According to the recommendation G.694.1 of ITU-T, in the flexible optical networks, optical spectrum is divided into subcarriers called also frequency slot. Each frequency slot has a central frequency $F_{c}$ (in THz) defined by $F_{c}=191.3 \times n+$ $0.125 ; n$ is relative numbers. Furthermore, the spectrum band assigned to a connection consists of several subcarriers which width is $S_{B}=12.5 \times m$ [4] with $m$ the number of frequency slots. One of the advantages of flexible optical networks is its capability to adapt the bandwidth defined by the number of frequency slot to the flow of traffic and modulation format according to the transmission distance. The formula in (1) indicates the number of frequency slots required by a connection of capacity $\mathrm{C}$ in $\mathrm{Gb} / \mathrm{s}$.

$m=\left\lceil\frac{C}{M \times F_{\text {slot }}}\right\rceil$

$M \times F_{\text {slot }}$ represents the capacity of a frequency slot in $\mathrm{Gb} / \mathrm{s} . \quad M(b / \mathrm{s} / \mathrm{Hz})$ is the modulation level in bits per second and represents the efficiency of the selected modulation format. $F_{\text {slot }}$ is the bandwidth of a frequency slot in GHz. M can take the values 1, 2, 3 or 4 depends on the modulation format is BPSK, QPSK, 8-QAM or 16-QAM [5]. The use of OFDM technology allows simultaneous transmission on parallel subcarriers. However, differential delays that have two sources in the flexible optical network must be taken into account[6]. The first source is the differential delay induced by Group Velocity Delay (GVD) which is related to the fact that the subcarriers of different frequencies have different transmission speeds, even when they are adjacent. That is to say; they form a monolithic block. Thus maximum differential delay caused by GVD related to $\mathrm{B}$ spectrum bands on a path length $\mathrm{L}$ is given by the formula in the equation (2.1).

$\Delta d_{\text {max }} \approx D\left(f_{c}\right) \times \sum_{1=1}^{B}\left(f_{\text {max }}-f_{\text {min }}\right) \times L$
Where $D\left(f_{c}\right)$ is the fiber dispersion at the level of central frequency, and $f_{\max }, f_{\min }$ represent respectively the maximum and minimum frequency of each spectrum band. Let's also note that the fiber dispersion of an SMF (Single Mode Fiber) is $17 \mathrm{ps} / \mathrm{nm} / \mathrm{km}$. The second source is the differential delay due to propagation delay and latency in the nodes. The propagation delay of the signal per kilometer is $r_{1}=5 \mu \mathrm{s} / \mathrm{km}$ and maximum latency in a node $r_{2}=25 \mu \mathrm{s} /$ $\mathrm{km}$. Thus the delay of transmission of traffic on a path $\mathrm{P}$ of distance $\mathrm{L}$ and having $\mathrm{N}$ links by adding the GVD is given by (2.2).

$$
D_{p}=L \times r_{1}+(N+1) \times r_{2}+\Delta d_{\max }
$$

And the formula (2.3) gives the transmission differential delay (DD) between two physical paths $P_{i}$ and $P_{j}$.

$$
D D=\left|D_{P_{i}}-D_{P_{j}}\right|
$$

According to ITU-G 709, the tolerable threshold of DD is $250 \mu \mathrm{s}$. But there are commercial products that achieve a tolerable DD up to128 ms by adding the SDRAM memory Off-Chip [8, 9].

\section{B. Spectrum allocation for time-varying traffic}

In the operational phase of the network, traffic is dynamic and fluctuates. There are essentially two types of dynamic traffic. There is in one hand, traffic of connections that have some finite amount of time, and their spectrum paths are released after this short time. The frequency slots belong to these spectrum paths become available for another connection. In the other hand, there is traffic of permanent connections whose traffic fluctuate. That kind of connections that corresponds more to reality, and we call them timevarying traffic connections. The traffic fluctuation of these type of connections translates to an additional demand for frequency slots in the case of traffic expansion or release of some frequency slots in traffic contraction. The policy of dynamically adapt the required frequency slots of a connection to traffic fluctuation is called Spectrum Expansion/Contraction (SEC) policy [10].

In this study, we assume that the request of frequency slots for each connection occurs at a rate $\lambda$ according to a Poisson process. Moreover, the duration follows an exponential distribution parameter $\mu$ equal to 1 . Therefore, the load of each connection under this fluctuation is $\lambda / \mu$. Based on the calculation of $\lambda$ in [11] with a fixed modulation format, the traffic load $\rho$ is obtained through the following: $\rho=\lambda=M \times m$, where $m$ is the number of frequency slots and $M$ the modulation efficiency of each modulation format, according to principle of "half law" [5]. The values respectively taken by $M$ can be 1, 2, 3 or 4 belong to BPSK, QPSK, 8-QAM or 16QAM modulations. A time-varying traffic connection has a reference frequency $\mathrm{F}_{\mathrm{R}}$ and a number $m_{t_{i}}$ of frequency slots allocated at time slot $t_{i}$. This number of frequency slots allocated to connection $R$ at the time slot $t_{i}$ is composed by $m_{t_{i}}^{H}$ and $m_{t_{i}}^{L}$ which are respectively a number of frequency slots used on the lower side and the upper side of the connection's reference frequency. That number $m_{t_{i}}=m_{t_{i}}^{H}+$ $m_{t_{i}}^{L}$ is assumed to be constant during each time slot $t_{i}$. Figure 
1 shows spectrum occupancy by connection $R$ and its neighboring connections.

The spectrum allocation to time-varying traffic connections as indicated by several studies $[10,12]$ is an ILP optimization problem. It is also known to be np-hard when the network size increases. Heuristics are most common in resolving this problem. These heuristics method of frequency slots allocation to time-varying traffic connections can be classified into two categories as follows: the expansion/ contraction frequency slots within the constraints defined in [10] and the combining of expansion/contraction with defragmentation or reconfiguration policies. The first studies of spectrum resources allocation to time-varying traffic connections deals with the methods of expansion/contraction of frequency slots, based on the constraint that two adjacent connections must not use the same frequency slots simultaneously in the same time slot. In this way, the paper [12] provides three policies of spectrum expansion/contraction depending on the fluctuation in traffic. These policies differ from one to other by the limitations suffered by the reference frequency and the allocated spectrum band width. The first of these is the fixed allocation in which the reference frequency and allocated spectrum band width remain fixed and reserved. This spectrum band is exclusively allocated to a particular connection and cannot be used by another connection. Even if, the full width of the spectrum band has is unused. That leads to a waste in case of contraction and request blocking when the request of the connection resulting from the expansion goes beyond the width of the allocated spectrum band. The second policy of spectrum expansion/contraction taking into account is called semi-elastic allocation, in this case, the reference frequency is fixed and can not be moved from its initial position. But, the spectrum bandwidth is allowed to extend or contract according to the traffic fluctuation and the limits of frequency slots occupied by the adjacent connections for each time slot. Frequency slots can be shared out between adjacent connections in different time slots, based on changes in traffic of connections. The third one is the elastic allocation that gives the way to move the reference frequency and extend or restrict the frequency slot block according to traffic variations of the connection. The elastic allocation allows more flexibility in the spectrum managing than the others mentioning above. Three other methods of SEC policies are developed in [10]. There are Constant Spectrum Allocation (CSA), Dynamic High-Low Expansion Contraction (DHL) and Dynamic Alternate Direction (DAD). In the CSA, physical path and a reference frequency are allocated to every connection.The connection has exclusive use of spectrum band located between the reference frequency and the reference frequency of the adjacent connection located above. This method does not allow the sharing out of frequency slots with adjacent connections, even if there are available. As for DHL, it enables the sharing out of frequency slots between adjacent connections. With DHL, a connection that needs to expand its flow first scans the frequency slots which are located on the higher side as its reference frequency to achieve frequency slots already used by the adjacent connection higher reference frequency. Then, if it needs additional frequency slots, it explores the frequency slots on the side below the reference frequency. Concerning to DAD, it also allows the sharing of frequency slots between connections. However, for the DAD, SEC takes place alternatively in both directions. These methods on-cited define the policy of expansion or contraction of the connections. According to fluctuations in traffic, the incease is limited by the constraint of non-overlapping. Formula (3) defines this constraint of non-overlapping that induces two inequalities.

$$
\begin{aligned}
& 0 \leq m_{t_{i}}^{H} \leq \min _{e}\left(F_{U_{e}}-m_{U_{e}}^{L}\right)-F_{R}-G, \text { and } \\
& 0 \leq m_{t_{i}}^{L} \leq F_{R}-\max _{e}\left(F_{B_{e}}+m_{B_{e}}^{H}\right)-G
\end{aligned}
$$

$U_{e}$ and $B_{e}$ represent the highest and, the lowest adjacent connections of connection $R$ on link $e$ that include in path $p$ of the network represented by a graph $(\mathrm{V}, \mathrm{E})$. $\mathrm{V}$ is the set of nodes which can be bandwidth variable transponders (BVTs) or Optical Cross-connect (OXCs) and $\mathrm{E}$ is the set of fiber links. $F_{B_{e}}$ and $m_{B_{e}}^{H}$ are respectively the reference frequency and the higher frequency slots used by connection $B_{e}$ on link $e . F_{U_{e}}$ and $m_{U_{e}}^{L}$ are also respectively the reference frequency and the lower frequency slots occupied by connection $U_{e}$ on link $e$. Figure 1 inspired by, thus, provides in [10] shows spectrum occupancy of this adjacent connection on links e and e'.

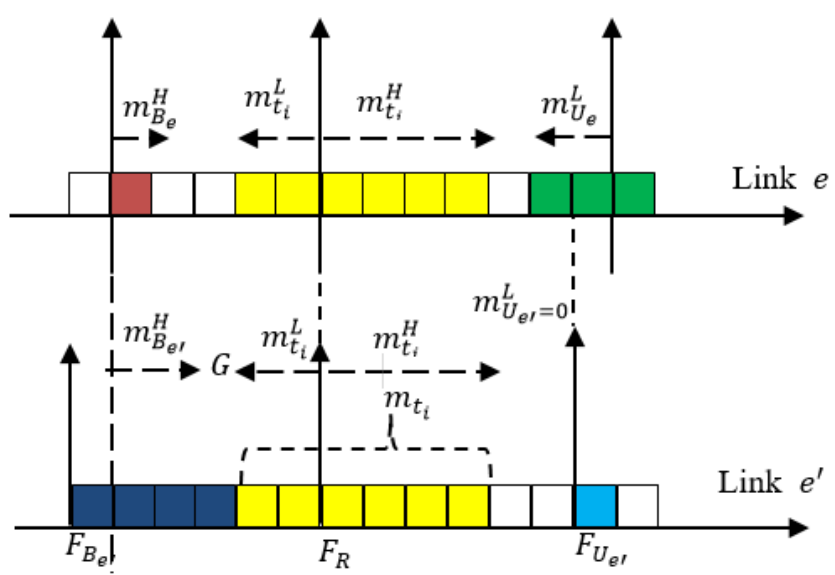

Fig. 1. Spectrum occupancy of connection $R$ and its neighbor connections $U_{l}$ and $B_{l}$ on two links e, e' at time $t_{i}$

The rise in transmission rate leads to the block of requests when the above SEC policies have been implemented. Even, if the sharing out of frequency slots among adjacent connections are allowed in the SEC policy, blocking will occur certainly when the connection, due to his traffic expansion has already used the available frequency slots in the both side of his reference frequency. In other words, when the equations (4.1) and (4.2) occur and the connection still needs additional frequency slots to satisfy traffic increase.

$$
\begin{gathered}
m_{t_{i}}^{H}=\min _{e}\left(F_{U_{e}}-m_{U_{e}}^{L}\right)-F_{R}-G \\
m_{t_{i}}^{L}=F_{R}-\max _{e}\left(F_{B_{e}}+m_{B_{e}}^{H}\right)-G
\end{gathered}
$$

To cope with this blocking of connections, defragmentations policies are implemented. Defragmentation consist in reallocating or rerouting currunt running connections to make enough spectrum paths for a new one or 
future arrival connections. These defragmentations policies explore other areas of the spectrum to release necesary contiguous and adjacent frequency slots required by traffic expansion [11]. The first one is the Shift Blocking Neighbors (SBN) which tries to reallocate the adjacent connections without touching the reference frequency. The second method of defragmentation is the Float Blocking neighbors (FBN), unlike the SBN provides the ability to move the reference frequency and connections, therefore, have the opportunity to float in the spectrum. When a new request arrives for a connection, in the absence of frequency slots in the expansion authorized area, the FBN tries to move the connections to have a maximum expansion zone. This tests initially to release the spectrum occupied by its neighbor connection in the direction that maximizes the minimum number of frequency slots available among all its neighbors. If this is not enough, so it tries to move the connection that is in the other direction. However, the approach based on defragmentation, even if it brings a significant reduction in the blocking rate is expensive for the operator because it can cause some interruption of existing connections or require the use of additional transponders. So, to avoid the reverse effects of defragmentation, we propose an allocation strategy based on the multi-spectrum bands.

\section{Multi-Spectrum BANDS Allocation In FleXiBle OPTICAL NETWORKS}

In this section, we propose an optimization model with the objective of minimization blocking rate. We introduce in this model the concept of multi-spectrum bands and constraint of différential delay, due to: GVD, propagation delay and node latency. We also take into account usually constraints in flexible optical network; those are contiguity, continuity and non-overlapping spectrum. As the model is np-hard; a heuristic method is proposed for solving it.

\section{A. Optimization model}

We address the problem of spectrum allocation to timevarying traffic connections with the distribution of traffic load during a specific time slot on multiple paths. This allocation have a priority objective for reducing the blocking rate. The blocking rate is calculated by dividing the number of rejected requests by the total number of requests. It is true that traffic suffers fluctuations during a certain period composed of time slots. We assume that each time slot $t$ demand is constant. Here, is the mixed integer linear programming model. The model relies on the concept of spectrum path which is set of a specific physical path composed by fiber links and spectrum band. Spectrum band is some amount of adjacent frequency slots. Before the optimization model, we give the notations and variables (Table 1).
TABLE I. NOTATIONS AND VARIABLES

\begin{tabular}{|c|c|c|}
\hline Symbol & & Description \\
\hline $\mathrm{G}(\mathrm{V}, \mathrm{E})$ & : & $\begin{array}{l}\text { A graph represents a flexible optical network with } \\
\text { nodes in set } \mathrm{V} \text { and edges in set } \mathrm{E} \text {. }\end{array}$ \\
\hline$e$ & : & $e \in E:$ link of fiber \\
\hline$s_{i}$ & : & A frequency slot with index $i$ \\
\hline $\mathrm{S}$ & : & Set of frequency slots \\
\hline$t_{i}$ & : & Time slot of period $T$ \\
\hline$P_{b}(T)$ & : & Blocking rate during period $T$ \\
\hline$N_{b}\left(t_{i}\right)$ & : & The number of requests blocked during time slot $t_{i}$ \\
\hline$N_{\text {tot }}\left(t_{i}\right)$ & : & Total number of requests during time slot $t_{i}$. \\
\hline$\Delta$ & : & Set of all permanent connections in traffic fluctuates \\
\hline $\mathcal{R}$ & : & A particular connection of $\Delta$ \\
\hline$D$ & : & $\begin{array}{l}\text { Set of all requests resulting from changes in } \\
\text { connections that belong to } \Delta \text { at time slot } t_{i} \text {. }\end{array}$ \\
\hline$d$ & : & A particular demand in $D$ \\
\hline $\mathcal{L}(d)$ & : & Set of allowable spectrum path of demand $d$ \\
\hline $\mathcal{L}(D)$ & : & $\begin{array}{l}\text { Set of all allowable spectrum path } \\
\mathcal{L}(D)=\bigcup_{d \in D} \mathcal{L}(d)\end{array}$ \\
\hline$\zeta$ & : & Number of allowable spectrum path in $\mathcal{L}(d)$ \\
\hline$\ell$ & : & A particular spectrum path in $\mathcal{L}(d)$ \\
\hline$d_{i}$ & $:$ & $\begin{array}{l}\text { A split of demand } d \text { on particular allowable spectrum } \\
\text { path } \ell\end{array}$ \\
\hline$m_{l}$ & : & Number of frequency slots allocated to sectrum path $\ell$ \\
\hline$p_{l}$ & : & Physical path of spectrum path $\ell$ \\
\hline$\left|p_{l}\right|$ & : & Number of link e in physical path $p_{l}$ \\
\hline$\eta_{\ell \ell}$ & : & Differential delay between spctrum path $\ell$ and $\ell^{\prime}$ \\
\hline$x_{d_{i} l}$ & : & $\begin{array}{l}\text { Boolean, equal to } 1 \text { if } d_{i} \text { use spectrum path } \ell \text {, else it } \\
\text { equals to } 0\end{array}$ \\
\hline$x_{l, l}$ & : & $\begin{array}{l}\text { Boolean variable equals to } 1 \text { if physical paths } p_{l} \text { and } \\
p_{l}{ }^{\prime} \text { share at leat one edge, else it is } 0\end{array}$ \\
\hline$x_{l, l}, e$ & : & $\begin{array}{l}\text { Boolean variable equals to } 1 \text { if physical paths } p_{l} \text { and } \\
p_{l}{ }^{\prime} \text { share the same edge } e \text {, else it is } 0\end{array}$ \\
\hline$x_{l, e}$ & : & $\begin{array}{l}\text { Boolean variable equals to } 1 \text { if physical path } p_{l} \text { used } \\
\text { edge } e \text {, else it is } 0\end{array}$ \\
\hline$x_{l, i}$ & $:$ & $\begin{array}{l}\text { Boolean variable equals to } 1 \text { if spectrum path } l \text { use } \\
\text { frequency slot } s_{i} \text { share at leat one edge, else it is } 0\end{array}$ \\
\hline
\end{tabular}

\section{Objective function}

Minimize

$$
P_{b}(T)=\sum_{t_{i} \in T} \frac{N_{b}\left(t_{i}\right)}{N_{t o t}\left(t_{i}\right)}
$$

Equation (5.1) is the objective function of the overall block rate during the period $\mathrm{T}$.

the following contraints must be met at every slot time.

\section{Constraints}

$$
d=\sum_{i} d_{i}
$$


Equation (5.2) is a constraint on the distribution of the flow of traffic during the time slot. Indeed, traffic distribution on the different spectrum paths should equal the overall flow traffic requested by a connection. A traffic demand of particular existent connection is split in several $d_{i}$ flows.

$$
\sum_{d i} \sum_{l \in \mathcal{L}(d)} d_{i} l \leq \zeta
$$

The constraint (5.3) indicates that the number of spectrum paths $l$ in which request $d$ is splitting must be lower or equal the spectrum path that respect the following differential delay constraint (5.4).

$$
\begin{aligned}
& \forall \ell, \ell^{\prime} \in \mathcal{L}(d): \eta_{\ell \ell^{\prime}} \leq \tau \\
& \sum_{e \in p_{l}} \sum_{i} x_{p_{l, e, i}}=\left|p_{l}\right| \times m_{l}
\end{aligned}
$$

Equation (5.5) expresses the constraint of contiguity or adjacency on each link of spectrum path. In each link, $m_{l}$ frequency slots forming the spectrum band must be contiguous. To ensure the continuity, the contiguity constraint of the same $m_{l}$ frequency slots must be met in all the $\left|p_{l}\right|$ links of the path $p_{l}$.

$$
\begin{aligned}
& \forall \ell, \ell^{\prime} \in \mathcal{L}(D), l \neq l^{\prime}, e \in E: x_{l, l^{\prime}} \leq \sum_{e} x_{l, l^{\prime}, e} \\
& \forall \ell, \ell^{\prime} \in \mathcal{L}(D), l \neq l^{\prime}, e \in E: x_{l, l^{\prime}, e} \leq x_{l, e} \\
& \forall \ell, \ell^{\prime} \in \mathcal{L}(D), l \neq l^{\prime}, e \in E: x_{l, l^{\prime}, e} \leq x_{l^{\prime}, e} \\
& \forall \ell, \ell^{\prime} \in \mathcal{L}(D), l \neq l^{\prime}, s_{i} \in S: x_{l, i}+x_{l i}+x_{l, l^{\prime}} \leq 2
\end{aligned}
$$

Constraints (5.6), (5.7), (5.8) and (5.9) ensure the nonoverlapping constraints. One frequency slot can not be used simultaneously by two different spectrum paths that share at least one link. The variable $x_{l, l^{\prime}}$ is a boolean that takes value 1 when spectrum paths $l$ and $l$ ' share at least one link, otherwise it takes value 0 . His value is determined by constraints (5.6), (5.7) and (5.8). As to the constraint (5.9), it ensures frequency slot $s_{i}$ can not be allocated to spectrum paths $l$ and $l^{\prime}$, if they have a link sharing. If frequency slot $s_{i}$ is used simultaneously by spectrum paths $l$ and $l^{\prime}$ then $x_{l, i}+x_{l^{\prime} i}+x_{l, l^{\prime}}=3$ or that is impossible because whatever values, this constraint (5.9) must be less than or equal to 2 .

This problem output is the set of spectrum paths allocated to a set of demands when permanent connections traffics fluctuate. As in each time slot, the novel request of the spectrum is constant. We can say that this problem can be seen as offline routing and spectrum assignment in each time slot [13]. So, this problem is np-hard. When the network size grows the time to find an optimal solution also grows exponentially as several studies have already shown it $[2,11$, 12]. In that condition, the suitable heuristic can be used to obtain near optimal solution within a reasonable time. With this end, in the following section, we shall propose a heuristic based on multi-spectrum bands concept in which it contributes to avoiding the worst effect when defragmentation or reconfiguration policies are used when blocking occur.

\section{B. Heuristic multi-spectrum bands allocation}

Our heuristic relies on the principle of combining the implementation of the DAD with the multi-spectrum band allocation. This concept of Multi-Spectrum Band Allocation (MSBA) means that traffic is progressively distributed over different spectrum paths. Each of these spectrum paths is formed by a physical path and a spectrum band so that the sum of all spectrum bands can support all traffic. After running the $\mathrm{DAD}$, in the case of dissatisfaction of the entire request, another optical path is used to bear the remaining traffic and so on until there is no traffic. As regards the distribution of traffic on the spectrum bands, each spectrum band used to carry some traffic must have a minimum width. This precaution must be taken to avoid a proliferation of spectrum bands taken into account the transmission of traffic. Indeed, the proliferation of spectrum bands causes waste of frequency slots. It is necessary to associate a guard band to reduce interference between different adjacent spectrum paths. For the allocation spectrum band to traffic supplement after the $\mathrm{DAD}$, we choose a spectrum path which allows allocating the entire traffic supplement. In the absence of such a path, we divide the traffic supplement on the k-shortest paths of the connection whose traffic fluctuates. We assumed that the kshortest paths of each connection are determined by Yen's algorithm [14] and an initial path with a reference frequency is associated with each connection. The modulation format used is chosen according to the transmission distance and follow the "half-distance low"[5]. The heuristic can been reflected by the following steps:

Step 1: Run the Dynamic Alternate Direction (DAD) as a policy of expansion/ contraction of the spectrum when the traffic of connection fluctuates.

\section{Step 2: In case of dissatisfaction,}

Select all the paths respecting differential delay constraints.If there is one which supports all the traffic, make the allocation on this path.

\section{Otherwise, go to step 3} band

Step 3: Select the first path that offers the largest spectrum

Step 4: Make the allocation of traffic supplement, if not the entire satisfaction. Return to Step 3 to find another path to allocate the remaining traffic. Repeat this until there is no more traffic or no more path.

The algorithm below presents the pseudo-code that reflects the MSBA heuristic. We assume that at the end of time slot $t_{i}$, traffic request for time slot $t_{i+1}$ is known. Here are the meaning of the variables of the pseudo-code:

- $C\left(t_{i+1}, s, d\right)$ : the flow of traffic required at time $t_{i+1}$ by the connection from the source $s$ to destination $d$.

- $S A\left(t_{i+1}, s, d\right)$ : the allocation of frequency slots to a connection at time $t_{i+1}$ from source $s$ to destination $d$.

- SD: number of frequency slots corresponding to traffic $C\left(t_{i+1}, s, d\right)$ after choosing an appropriate modulation format. 
- SDAD: Number of frequency slots available around the reference frequency.

- $C_{r}\left(t_{i+1}, s, d\right)$ : Traffic flow remaining after execution of the Dynamic Alternative Direction.

- SSUP: additional frequency slots corresponding to $C_{r}\left(t_{i+1}, s, d\right)$

- SDISP: frequency slots available on a particular path $P_{\text {diff }}$

- DAD: Dynamic Alternative Direction

- $\quad P_{\text {diff }}$ : The subset of k-shortest path connecting that respect the differential delay.

\begin{tabular}{|c|c|}
\hline \multicolumn{2}{|c|}{ Algorithm of MSBA heuristic } \\
\hline Input: & $\begin{array}{l}\text { Network } \mathrm{G}(\mathrm{V}, \mathrm{E}), \text { Set } \Delta \text { of connections } \\
\mathrm{SA}\left(\mathrm{t}_{\mathrm{i}}, \mathrm{s}, \mathrm{d}\right) \\
C\left(t_{i+1}, s, d\right)\end{array}$ \\
\hline Output & $S A\left(t_{i+1}, s, d\right)$ \\
\hline \multicolumn{2}{|l|}{ BEGIN } \\
\hline 1: $\quad S$ & $S D \leftarrow \frac{C\left(t_{i+1}, s, d\right)}{M \times F_{\text {slot }}}$ \\
\hline 2: & Run dynamic alternative direction SEC policy \\
\hline 3: & $C_{r}\left(t_{i+1}, s, d\right) \leftarrow(S D-S D A D) \times M \times F_{\text {slot }}$ \\
\hline 4: & While $\left(C\left(t_{i+1}, s, d\right) \neq 0\right.$ and $\left.P_{\text {diff }} \neq \varnothing\right)$ do \\
\hline 5: & For all path in $P_{\text {diff }}$ do \\
\hline 6: & $\begin{array}{l}\text { SSUP } \leftarrow \frac{C_{r}\left(t_{i+1}, s, d\right)}{M \times F_{\text {slot }}} \\
\text { If SSUP is available in current } P_{\text {diff }} \text { path then }\end{array}$ \\
\hline $\begin{array}{l}\text { 8: } \\
\text { 9: }\end{array}$ & $\begin{array}{l}\text { Assign spectrum on this path to the } \\
\text { connection } \\
\text { Break for }\end{array}$ \\
\hline 10 & Else \\
\hline 11: & $\begin{array}{l}\text { Select among the path in } P_{\text {diff }} \text { the one } \\
\text { which have the highest SDISP }\end{array}$ \\
\hline $\begin{array}{l}\text { 12: } \\
\text { 13: }\end{array}$ & $\begin{array}{l}\text { Assign spectrum with size SDISP on this } \\
\text { path }\end{array}$ \\
\hline 14: & $\begin{array}{l}\text { Extract this path from } P_{\text {diff }} \text { set } \\
C_{r}\left(t_{i+1}, s, d\right) \leftarrow(S S U P-S D I P) \times M \times F_{\text {slot }}\end{array}$ \\
\hline
\end{tabular}

\section{5: $\quad$ End for}

\section{6: $\quad$ End while}

17: $\quad$ If $C_{r}\left(t_{i+1}, s, d\right)=0$ then spectrum assignment is

18: $\quad$ accomplished

END

Else Spectrum assignment is blocked

\section{Simulation AND ANALysis OF Results}

The simulations to evaluate the performance of our proposal were carried out on two network topologies. The NSFNET network (14 knots, 22 links) well-known network is using to simulate most of the work in this area. The second network is the national Backbone of Ivory Cost from the will of the State of Ivory Cost to mesh all Ivorian territory with a fiber optical transport (28 nodes and 46 links) as shown in Figure 2.
A guard band is $G=1$ slot, and as regards the choice of modulation format, each path has a format according to the principle of "half distance law" [5]. Furthermore, we assume that each connection is associated with a reference frequency and 4-shortest paths determined by the Yen's algorithm [14]. It takes into account the initial path in which the connection is running before the fluctuation in traffic occurs. The differential delay is set at $\tau=250 \mu$ s as recommended by the ITUT.709. As for the distribution of traffic load, the minimum number of frequency slots corresponding to an eligible distribution is three frequency slots. It means the minimum width of spectrum band is three frequency slots. As for the dynamic traffic, each connection frequency slots request follows the Poisson process with a transmission flow $\lambda$ and duration which has an exponential distribution parameter $\mu=$ 1 as described in (Section II.B).

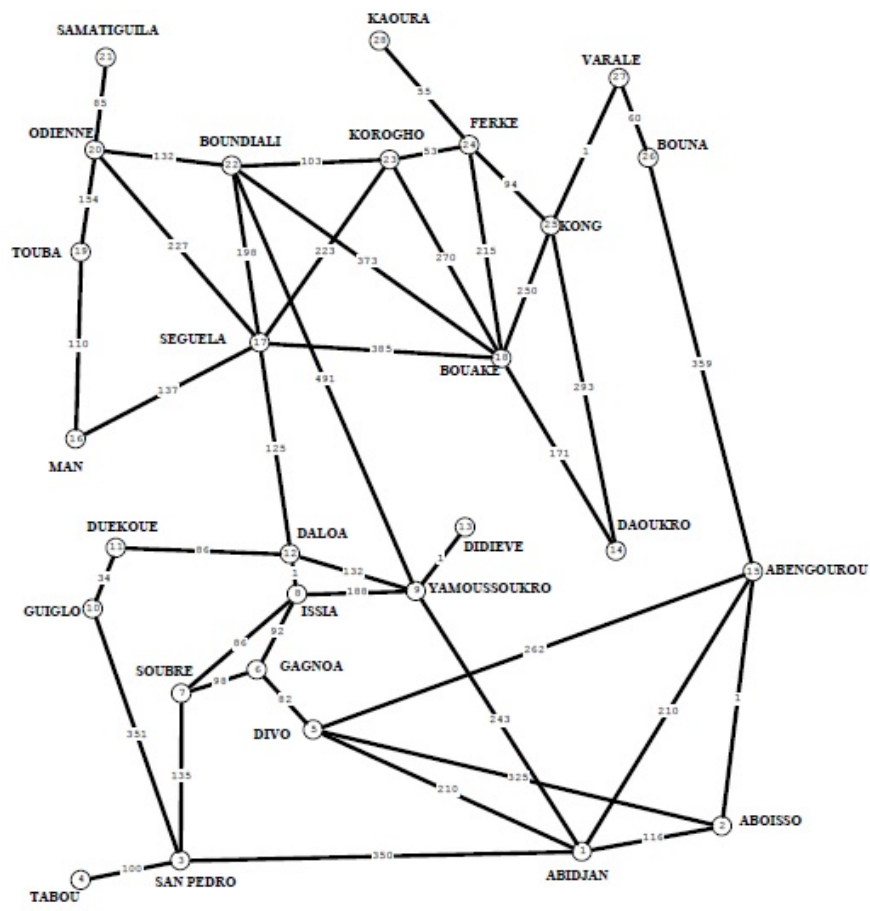

Fig. 2. Ivory Coast National Backbone Network

The simulations carried out in Java on a PC with a Dual Core processor (2.16 GHz) and memory 4GB. The aim was to observe the impact of traffic load and spectrum available on the blocking rate. Dealing with the impact of the traffic load, we vary the load from 200 erlangs (E) to $1600 \mathrm{E}$ by keeping the number of frequency slots per link at 100 frequency slots. Then, we evaluate its effect on the blocking rate. As for the impact of the number of frequency slots available by link, we vary the number of frequency slots from 100 to 240 frequency slots maintaining the traffic load at $1000 \mathrm{E}$.

The observed results are in the graphs of Figures 3 and 4 . Figure 3(a) shows the performance of the Dynamic Alternate Direction policy (DAD), Float Blocking neighbors (FBN), and our Multi-Spectrum Band Allocation policy (MSBA) on the blocking rate depending on the network load for the topology NSFNET. 

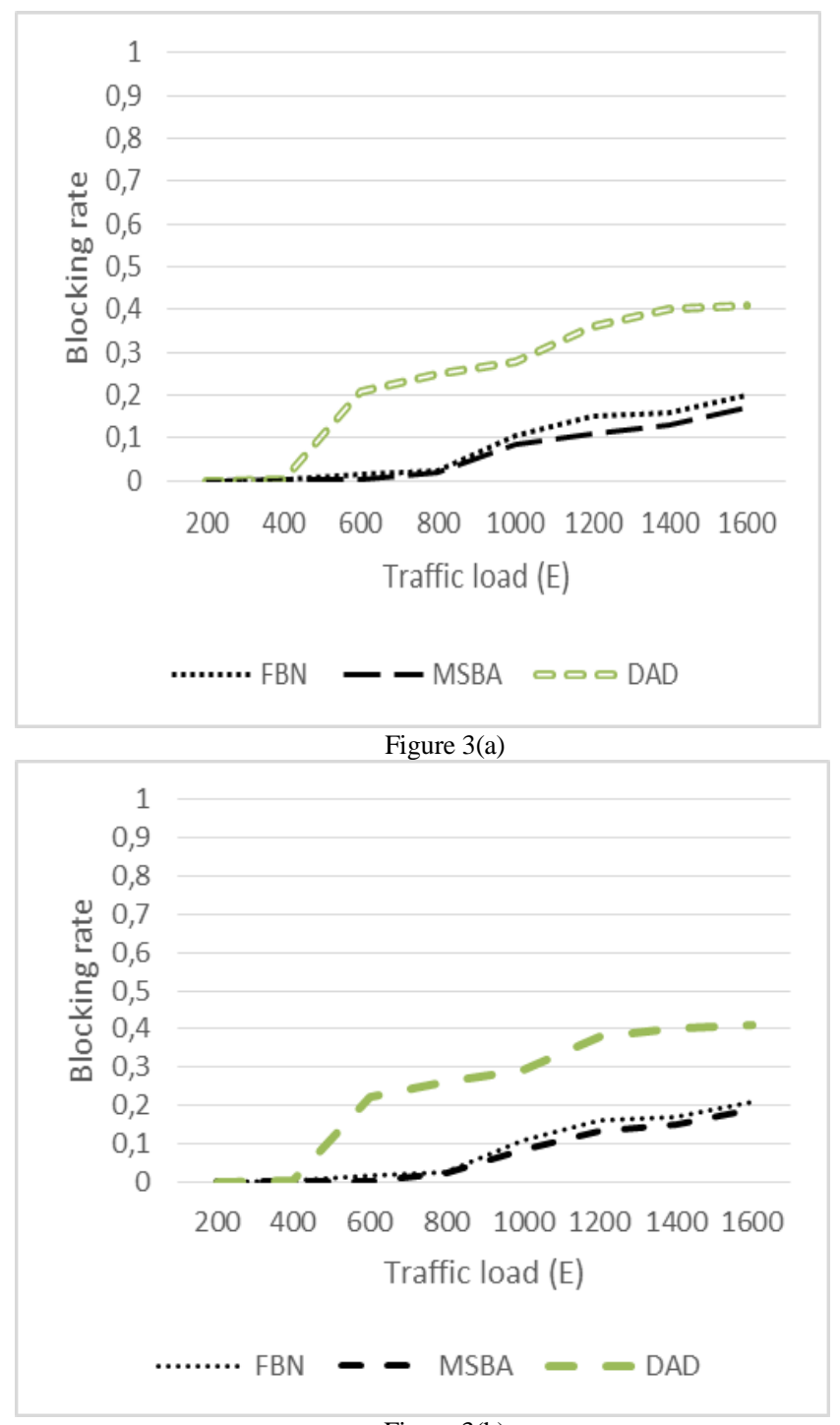

Figure 3(b)

Fig. 3. (a) Blocking rate as a function of Traffic load in the NSFNET. (b) Blocking rate as function of Traffic load in the Ivorian National Backbone

The number of frequency slots per fiber equal to $\delta=100$ when the network load varies from 200 to 1600 E. The MSBA has better performance than the DAD from 400E. But, regarding the FBN, method based on defragmentation, gives the same rates of blockages like MSBA under 1000E. After reaching $1000 \mathrm{E}$, slight improvement of the blocking rate is noted.

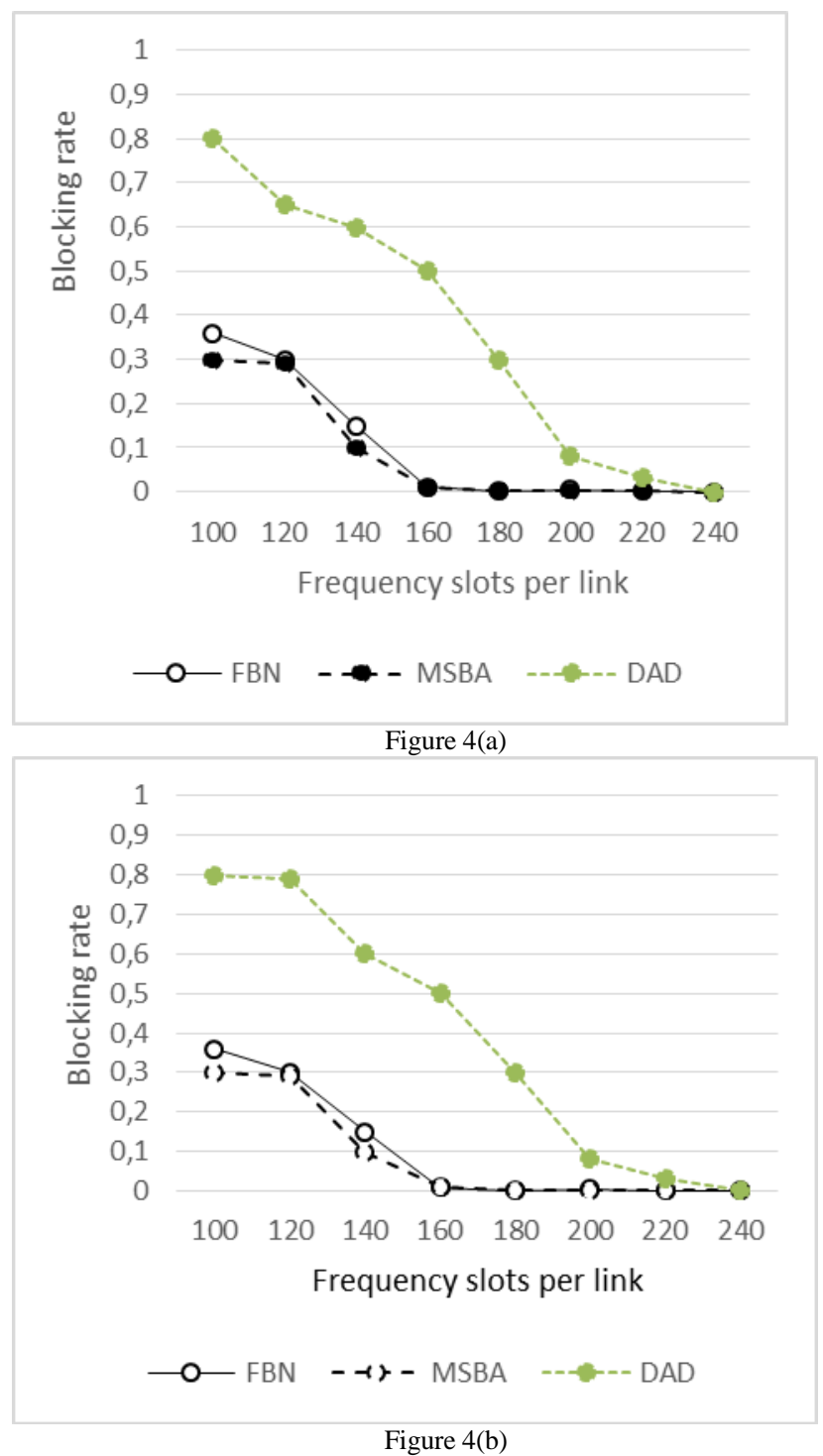

Fig. 4. (a) Blocking rate as a function of available slots in the NSFNET. (b) Blocking rate as a function of available Fiber link of the Ivorian National Backbone

Figure 3 (b) reproduces the same trends observed with the NSFENET topology on another national backbone topology Ivory Coast. In these simulations, it appears that MSBA reduces the blocking rate of about $16 \%$ by varying the traffic load than DAD method. Regarding the performance of MSBA compared to FBN, we remark a reduction in the blocking rate of about $3 \%$. 
Figure 4 (a) shows the performance of the Dynamic Alternate Direction (DAD), Float Blocking neighbors (FBN), and Multi-Spectrum Bands Allocation method (MSBA) on the block rate, based on the number of frequency slots with NSFNET topology. It sets the total traffic load to 1000 Erlangs. The number of link frequency slots varies from 100 to 240 frequency slots. As expected, the blocking rate decreases when the number of frequency slots increases. Also, compared to other methods MSBA provides a blocking rate significantly lower than the DAD. Nevertheless, beyond 160 frequency slots. The MSBA and FBN have almost the same performance. We observe the same trends with the national Ivory Coast backbone topology.

\section{CONCLUSION}

In this work, we have dealt with the problem of dynamic allocation of frequency slots in the flexible optical networks. The situation tackles the connections that have undergone fluctuation. We have offered a heuristic based on the distribution of the additional traffic load on strips of different spectrum paths. This method has simulated on the NSFNET network and Ivorian National Backbone topologies, allows a far interesting reductions on blocking rate when the fluctuation of traffic demand leads to an additional frequency slots. MSBA heuristic exploits the advantages of flexibility of modulation offered by flexible optical networks. Moreover, it appears as an interesting policy of blocking reduction for time-varying traffic connections. By avoiding harmful effects related to defragmentation method that often bring about the interruption of existing connections.

Our future reflections will focus on the recurring character of the traffic fluctuations, that has been observed in certain types of traffic, for instance Internet traffic; one can introduce predictions methods in spectrum allocation knowing the recurrence of traffic.

\section{REFERENCES}

[1] JINNO, Masahiko, TAKARA, Hidehiko, KOZICKI, Bartlomiej, et al. Spectrum-efficient and scalable elastic optical path network: architecture, benefits, and enabling technologies. Communications Magazine, IEEE, 2009, vol. 47, no 11, p. 66-73.
[2] LINKOWSKI, Miroslaw et WALKOWIAK, Krzysztof. Routing and spectrum assignment in spectrum sliced elastic optical path network. IEEE Communications Letters, 2011, vol. 15, no 8, p. 884-886.

[3] ZANG, Hui, JUE, Jason P., MUKHERJEE, Biswanath, et al. A review of routing and wavelength assignment approaches for wavelength-routed optical WDM networks. Optical Networks Magazine, 2000, vol. 1, no 1, p. 47-60.

[4] ITU-T, Spectral grids for wdm applications:Dwdm frequency grid,Recommendation G.694.1,Februry 2012

[5] A. Bocoi, M. Schuster, F. Rambach, M. Kiese, C.-A Bunge, and B. Spinnler, "Reach-dependent capacity in optical networks enabled by OFDM," OFC/NFOEC 2009, Paper OMQ4, March 2009.

[6] CHEN, Xiaomin, JUKAN, Admela, et GUMASTE, Ashwin. Optimized parallel transmission in elastic optical networks to support high-speed Ethernet.Journal of Lightwave Technology, 2014, vol. 32, no 2, p. 228238.

[7] Interfaces for the optical transport network (OTN),"ITU-T Recommendations.[Online].Available:http://www.itu.int/rec/T-RECG.709/e

[8] Intelixf19301datasheet.”[Online].Available:http://www.intel.com/ design/network/products/optical/tsp/IXF19301.htm

[9] Cisco xsl-series.[Online].Available:http://www.cisco.com/en/US/products/hw/modules/ps2710/ps5479/index.html.

[10] CHRISTODOULOPOULOS, Konstantinos, TOMKOS, Ioannis et VARVARIGOS, Emmanouel. Time-varying spectrum allocation policies and blocking analysis in flexible optical networks. Selected Areas in Communications, IEEE Journal on, 2013, vol. 31, no 1, p. 1325

[11] STIAKOGIANNAKIS, Ioannis, PALKOPOULOU, Eleni, KLONIDIS, Dimitrios, et al. Dynamic cooperative spectrum sharing and defragmentation for elastic optical networks. Journal of Optical Communications and Networking, 2014, vol. 6, no 3, p. 259-269

[12] KLINKOWSKI, Miroslaw, RUIZ, Marc, VELASCO, Luis, et al. Elastic spectrum allocation for time-varying traffic in flexgrid optical networks. IEEE journal on selected areas in communications, 2013, vol. 31, no 1, p. 26-38.

[13] CHATTERJEE, Bijoy Chand, SARMA, Nityananda, et OKI, Eiji. Routing and spectrum allocation in elastic optical networks: a tutorial. IEEE Communications Surveys \& Tutorials, 2015, vol. 17, no 3, p. 1776-1800.

[14] YEN, Jin $Y$. Finding the $k$ shortest loopless paths in a network. management Science, 1971, vol. 17, no 11, p. 712-716. 